\title{
Anti-PD-L1/TGFbetaRII Fusion Protein M7824
}

National Cancer Institute

\section{Source}

National Cancer Institute. Anti-PD-L1/T GFbetaR/I Fusion Protein M7824. NCI Thesaurus. Code C124229.

A bifunctional fusion protein composed of avelumab, an anti-programmed death ligand 1 (PD-L1) human monoclonal antibody, bound to the soluble extracellular domain of human transforming growth factor beta (T GFbeta) receptor type II (T GFbetaRII), with potential antineoplastic and immune checkpoint modulating activities. Upon administration, the TGFbetaRII moiety of M7824 binds to and neutralizes TGFbeta while the avelumab moiety simultaneously binds to PD-L1. This prevents TGFbeta- and PD-L1-mediated signaling, and increases natural killer (NK) cell and cytotoxic T-lymphocyte (CTL) activities. This inhibits tumor cell proliferation in susceptible tumor cells. TGFbeta and PD-L1 are both upregulated in certain types of cancers; their overexpression is associated with increased evasion of immune surveillance and contributes to poor prognosis. 